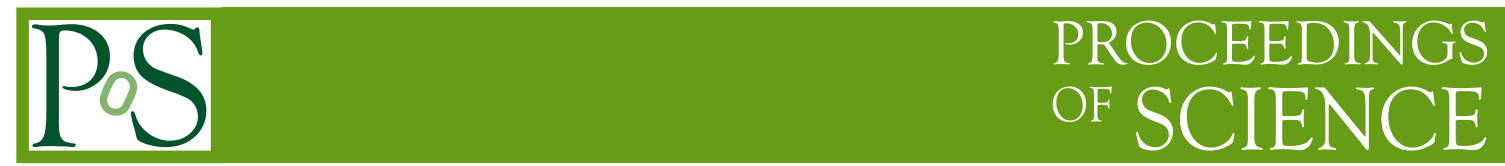

\title{
Search for a fermiophobic Higgs Particle
}

\author{
Matteo Sani* \\ On behalf of the CMS Collaboration \\ University of California, San Diego \\ E-mail: matteo.sani@cern.ch
}

\begin{abstract}
Vector boson fusion and associate production of Higgs bosons are searched for, with the Higgs decaying into vector bosons. The results are interpreted in a model for a fermiophobic Higgs. The full data sample of about $5 \mathrm{fb}^{-1}$ of pp collisions collected in 2011 with the CMS experiment at a CM energy of $7 \mathrm{TeV}$ have been analyzed, as well a significant fraction of the 2012 luminosity delivered so far at the new energy of $8 \mathrm{TeV}$.
\end{abstract}

36th International Conference on High Energy Physics,

July 4-11, 2012

Melbourne, Australia

\footnotetext{
* Speaker.
} 


\section{Introduction}

Several extensions of the Standard Model (SM) with an enlarged scalar sector have been proposed [1]. It is possible that the electroweak symmetry breaking mechanism responsible for generating the masses of the gauge bosons is independent of the mechanism that generates the fermion masses. In this case there should exist a Higgs boson that, at tree level, couples only to $\mathrm{W}$ and $\mathrm{Z}$ bosons. In the literature this model is usually referred to as the fermiophobic (FP) Higgs. Its decay to $\mathrm{W}$ and $\mathrm{Z}$ bosons is SM-like, while the decay to photons is via $\mathrm{W}$ loops. In the framework of the FP model, the branching ratio (BR) into two photons for a low mass Higgs boson $\left(\mathrm{M}_{H} \approx 120 \mathrm{GeV}\right)$ is enhanced by an order of magnitude with respect to the SM [2].The FP model Higgs has the SM-like production cross section for vector boson fusion (VBF) and associated production with a $\mathrm{W}$ or $\mathrm{Z}(\mathrm{VH})$, while gluon fusion and top quark fusion are absent or have a negligibly small cross section. Previous searches at LEP [3] and at the Tevatron [4] ruled out a FP Higgs boson lighter than $119 \mathrm{GeV}$ at $95 \%$ confidence level (CL). This paper describes the search for a FP model Higgs boson in $\gamma \gamma, \mathrm{WW}$ and ZZ decay modes [5] [6] in proton-proton collisions at a centre of mass energy of $7 \mathrm{TeV}$ and a relevant fraction of the 2012 luminosity delivered at the new energy of $8 \mathrm{TeV}$ at LHC with data collected by the CMS detector [7].

\section{Event Classes and Analyses}

Three decay channels of FP Higgs boson have been analyzed. The sensitivity of each analysis is increased by splitting the events in different classes according to their production mechanism. In the following a description of event classes and statistical treatment used in $\gamma \gamma$, WW and ZZ channels are given.

\section{$2.1 \gamma \gamma$ event classes}

Event selection of the $\gamma \gamma$ channel follow closely the one described in detail in the SM paper [8] where two isolated photon candidates with $\mathrm{p}_{T}>\mathrm{m}_{\gamma \gamma \mid} / 4, \mathrm{~m}_{\gamma \gamma} / 3$ and within the ECAL fiducial region $(|\eta|<2.5$ and $1.442<|\eta|<1.56)$ are required. Photons are identified by looking at the shape of electromagnetic shower and requiring that no track is associated with it to veto against electron. Also isolation variables are used as a mean to reject the non-prompt background due to electromagnetic showers originating in jets, mainly due to single and multiple $\pi^{0}$. Corrections for the additional effect caused by the multiple collisions in each bunch crossing (pile-up) are applied. Converted and unconverted photons are discriminated using the R9 variable, defined as the energy sum of $3 \times 3$ crystals centered on the most energetic crystal in the supercluster divided by the energy of the supercluster.

A class of $\gamma \gamma$ events that includes any event passing a dijet tag is defined to select Higgs bosons produced by the VBF process. Candidate diphoton events for the dijet-tagged class have the same selection requirements imposed on the photons as in the SM search [8] except for different $\mathrm{p}_{T}$ thresholds. The selection requirements have been optimized using simulated events, of VBF signal and diphoton background, to minimize the expected limit at 95\% CL on the VBF signal cross section. 
For a Higgs boson having a mass, $\mathrm{M}_{H}$ of $120 \mathrm{GeV}$ the overall acceptance times selection efficiency of the dijet tag for Higgs boson events is $15 \%$ for those produced by VBF, which corresponds to about 20.8 events. In this class of events, the expected signal-to-background ratio is close to one.

A class of events passing a lepton tag is aimed at selecting Higgs bosons produced by the $\mathrm{VH}$ process where the vector boson decays into an electron or a muon. Candidate diphoton events for the lepton-tagged channel have the same selection requirements imposed on the photons as in the $\mathrm{SM}$ search [8] except for the $\mathrm{p}_{T}$ thresholds which are different. Leptons are required to be separated from the photons by imposing a requirement on the angular distance between the lepton and each photon and are identified using the recommended identification criteria used in CMS analysis. To protect against the background events that arise from an electron misidentified as a photon in the $\mathrm{SM}$ process $\mathrm{Z} \rightarrow$ ee are required to have the invariant mass of photon-electron system at least $5 \mathrm{GeV}$ away the nominal mass of the $\mathrm{Z}$ boson. For each muon and electron class of the lepton-tagged channel the expected background yield is about 0.5 events in the $\mathrm{M}_{H}$ range $117.5 \div 122.5 \mathrm{GeV}$.

Significant fraction of signal events from decays of the vector bosons in $\mathrm{VH}$ production has characteristic signature with large missing transverse energy due to neutrinos escaping the detector (predominantly from $\mathrm{Z} \rightarrow v v$ decay). Events passed the lepton tag selections are excluded, so a statistically independent search is performed on $\mathrm{E}_{T}^{\text {miss }}$-tag events. Only photons detected in barrel, i.e. $|\eta|<1.5$, are included in the selection due to negligible contribution to the expected exclusion limit of the endcap photons. In addition $\mathrm{E}_{T}^{\text {miss }}$ is required to be larger than $70 \mathrm{GeV}$.

As the majority of the signal events are not expected to belong to the previous classes an untagged class of events is used to perform a statistically independent search. This class contains diphoton events that pass the inclusive $\gamma \gamma$ selection used in the SM search [8] but donÕt pass the exclusive selections described above. The Higgs bosons produced by VBF and VH mechanisms have a harder transverse momentum spectrum than those of the photon pairs produced by the background processes. To reduce the background while retaining high signal efficiency, a requirement on the ratio of transverse momentum and invariant mass of the two photons, $\pi_{T}^{\gamma \gamma}=\mathrm{p}_{T}^{\gamma \gamma} / \mathrm{m}_{\gamma \gamma}$ is imposed to define this untagged diphoton sub-channel. The expected S/B in this sub-channel is small, so events are further divided into four classes according to the expected mass resolution and signal purity using two photon classifiers: the minimum R9 of the two photons, and the maximum pseudorapidity of the two photons.

\subsection{WW and $\mathrm{ZZ}$ event classes}

\subsubsection{WW event classes}

The $\mathrm{H} \rightarrow \mathrm{WW} \rightarrow 212 v$ analysis [9], for the FP scenario, searches for an excess of events with two isolated leptons of opposite charge, large $\mathrm{E}_{T}^{\text {miss }}$ and two jets with VBF topology (diet class). The so-called "WW level" set of selections suppress the dominant background coming from top-quark production. Additional background contribution, mainly from Drell-Yan events, is suppressed by means of selections on the dilepton system mass and momentum, as well as on the angle between the dilepton and the dijet system. An optimized selection on the kinematic variables depending on the Higgs boson mass hypothesis is then applied. After all cuts, between 10 and 20 events, depend- 
ing on the Higgs boson mass hypothesis, remain in the signal regions. All the main backgrounds are estimated from the data.

The $\mathrm{WH} \rightarrow \mathrm{WWW} \rightarrow 313 v$ analysis instead searches for an excess of events with three leptons, electrons or muons, large missing transverse energy, and low hadronic activity (lepton class). The dominant background comes from $\mathrm{WZ} \rightarrow 31 v$ production, which is largely reduced by requiring that all same-flavor oppositely charged lepton pairs have a dilepton mass away from $\mathrm{M}_{\mathrm{Z}}$. After all cuts 7 events remain in the signal region.

\subsection{2 $\mathrm{ZZ}$ event classes}

In the $\mathrm{H} \rightarrow \mathrm{ZZ}^{(*)} \rightarrow 41$ channel [10], we search for a four-lepton mass peak over a small continuum background. The $4 \mathrm{e}, 4 \mu, 2 \mathrm{e} 2 \mu$ sub-channels are analyzed separately since there are differences in the four-lepton mass resolutions and the background rates arising from jets misidentified as leptons. The dominant irreducible background in this channel is from non-resonant $\mathrm{ZZ}$ production and is estimated from simulation. The smaller reducible backgrounds with jets misidentified as leptons, e.g. $\mathrm{Z}+$ jets, are estimated from data. In this case the results of SM Higgs search in ZZ channel have been just re-interpreted in the context of the FP Higgs benchmark model.

\subsection{Signal and Background Modeling}

To evaluate the compatibility of the data with the FP Higgs hypothesis, an extended likelihood function based on sum of analytic probability density functions (p.d.f) for the signal and background shapes is constructed.

For all the $\gamma \gamma$ event classes the invariant diphoton mass is used as a physical observable. In the untagged event classes only, an additional observables $\left(\pi_{T}^{\gamma \gamma}\right)$ is used and 2-dimensional models are constructed for both the signal and the background. The signal mass model is derived using Monte Carlo events while the background model is derived directly from data when possible. The choice of the functions used to fit the background is based on a study of the possible bias introduced on both the limit in the case of no signal and the measured signal strength in the case of a signal.

For the WW classes, the hypothesis testing is based on the number of expected signal and background events only, and therefore the likelihood function is built with Poissonian statistics. The number of expected signal events is evaluated from simulation, while the background contamination in the signal regions are estimated in a data-driven fashion whenever is possible.

For the $\mathrm{ZZ}$ classes the limit calculation is based on the same likelihood as for the $\gamma \gamma$ cases where the signal shapes are described by means of analytical fits and the background shapes are extracted from date when possible.

\section{Results}

The statistical approach considered in evaluating the limit is the asymptotic CLs approach using the profile likelihood ratio as a test statistic [11]. The search is carried out with steps of $0.5 \mathrm{GeV}$ in the signal hypothesis mass in range between 110 and $300 \mathrm{GeV}$. All known sources of systematic uncertainties are included in the likelihood model which is used for the limit setting. Systematic errors which are correlated between event classes (theory, luminosity, photon and trigger efficiency, etc) are included as common nuisance parameters in the likelihood model. 

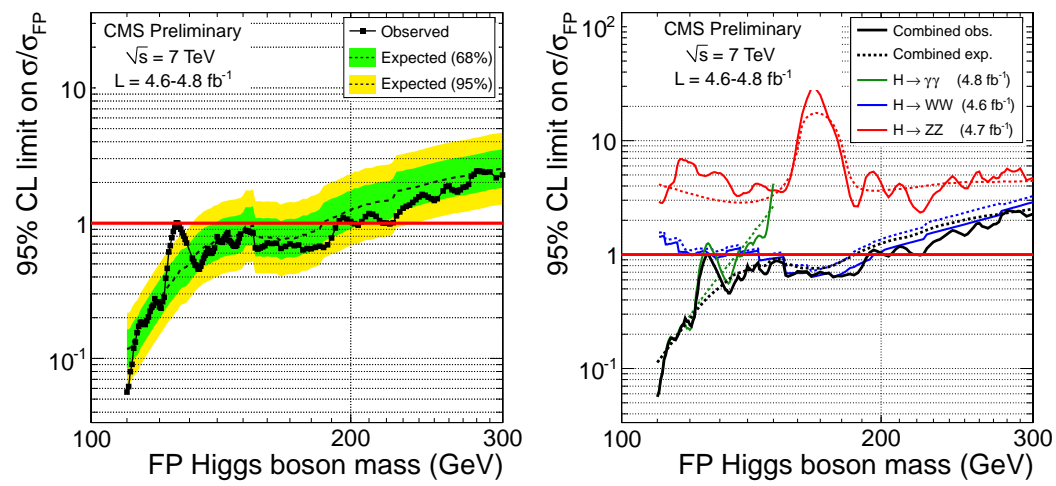

Figure 1: The observed and expected 95\% CL upper limits on the signal strength parameter $\mu=\sigma / \sigma_{F P}$ for the fermiophobic Higgs boson hypothesis as a function of the Higgs boson mass (left). The observed and expected 95\% CL upper limits on the signal strength parameter $\mu=\sigma / \sigma_{F P}$ as a function of the fermiophobic Higgs boson mass for the three explored decay modes and their combination (right).

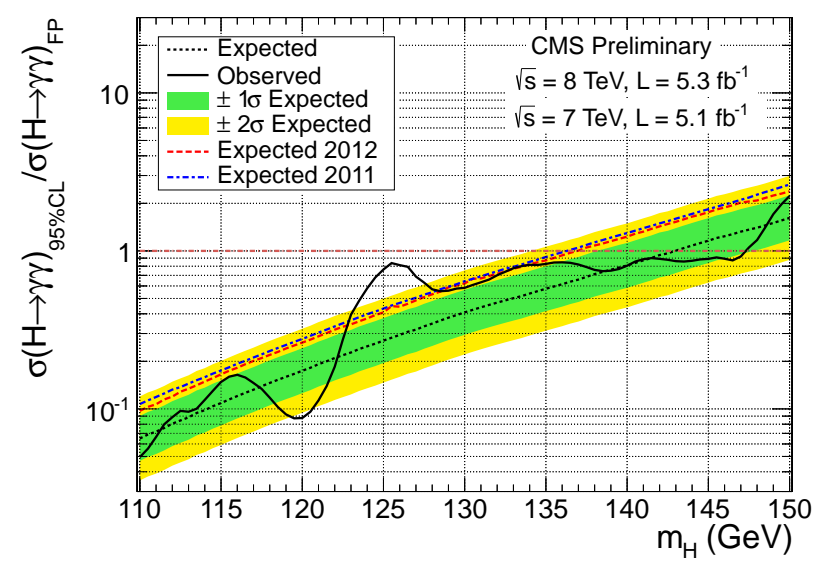

Figure 2: The 95\% CL combined 2011 and 2012 upper limit on the signal strength $\sigma / \sigma_{F P}$ of a FP Higgs boson, in the $\gamma \gamma$ decay mode as a function of the Higgs boson mass. Individual contributions to the expected limit for each period are shown in dotted lines.

Fig. 1 (left) shows the upper limit relative to the FP model expectation as a function of Higgs mass combining all three decay modes considered, $\gamma \gamma$, WW, and ZZ, using 2011 data at $7 \mathrm{TeV}$ centre of mass energy. The observed values (solid line) and the median of the expected results for the background-only hypothesis (dashed lines) are shown. The fermiophobic Higgs boson is excluded at $95 \% \mathrm{CL}$ in the mass range $110 \div 192 \mathrm{GeV}$. Also it is excluded at $99 \% \mathrm{CL}$ in the range $110 \div 188 \mathrm{GeV}$, with the exception of two gaps: $124.5 \div 128 \mathrm{GeV}$ and $148 \div 154 \mathrm{GeV}$. The contribution of the different channels to the exclusion limit is shown in Fig. 1 (right).

The $\gamma \gamma$ decay mode results have been updated analyzing $5.3 \mathrm{fb}^{-1}$ of data collected in 2012 at $8 \mathrm{TeV}$ centre of mass energy. The exclusion limit combining 2011 and 2012 data for this channel is shown in Fig. 2. The contribution to the expected limit of each of $\gamma \gamma$ sub-channel is shown as well. The FB Higgs boson is excluded at $95 \% \mathrm{CL}$ in the mass range $110 \div 147 \mathrm{GeV}$, at $99 \% \mathrm{CL}$ in the range $110 \div 134 \mathrm{GeV}$. 

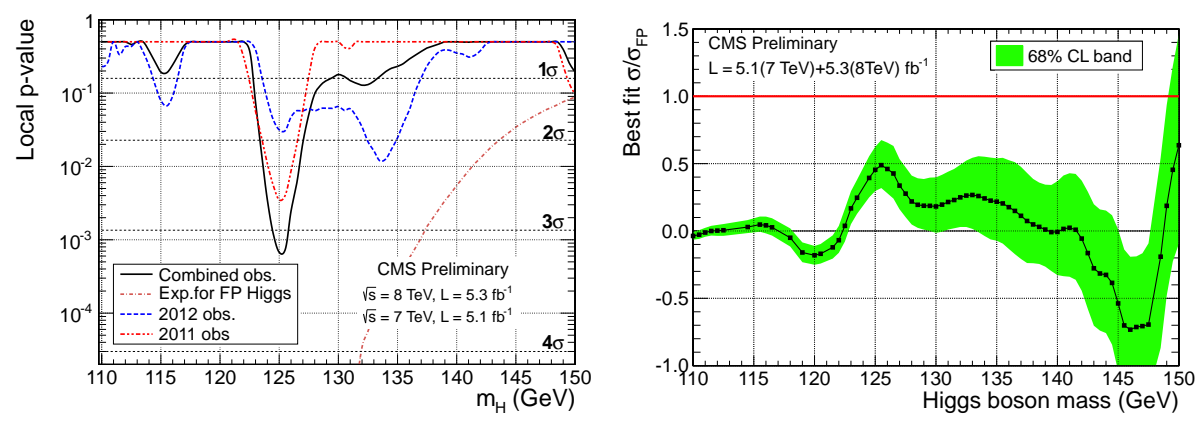

Figure 3: Combined 2011 and 2012 asymptotic p-value as a function of the Higgs boson mass for $\gamma \gamma$ decay mode only (contributions of each period is also shown) (left) and the best-fit value of the signal strength $\sigma / \sigma_{F P}$ for the combined sub-channels, as a function of the mass of a fermiophobic Higgs boson (right).

The largest upward fluctuation, at $125.5 \mathrm{GeV}$, has a global significance of $3.2 \sigma$. However this deviation is too weak to be consistent with the fermiophobic hypothesis as the observed signal strength for a FP Higgs boson is $0.49 \pm 0.18$. Fig. 3 show the local p-value with 2011 and 2012 contributions drawn separately (left) and the signal strength for a FP Higgs boson as a function of mass hypothesis (right).

\section{References}

[1] S.Mrenna and J. Wells (2000), arXiv:hep-ph/0001226.

[2] J. Gunion, H. Haber, G. Kane et al., "The Higgs Hunter's Guide". Addison-Wesley, 1989. For an early discussion of this model, see H.E. Haber, G.L. Kane, and T. Sterling, Nucl. Phys. B161, 493 (1979).

[3] ALEPH, DELPHI, L3, OPAL Collaborations, and The LEP Working Group for Higgs Boson Searches, "Search for Higgs bosons decaying into photons: Preliminary combined results using LEP data collected at energies up to $209 \mathrm{GeV}$ ", arXiv:0107035.

[4] CDF, D0, "Combined CDF and D0 upper limits on Fermiophobic Higgs Boson Production with up to $8.2 \mathrm{fb}-1$ of ppbar data", arXiv:1109.0576.

[5] CMS Collaboration, "Search for the Fermiophobic Model Higgs boson in pp collisions at sqrt(s) $=7$ TeV", DOI: 10.1007/JHEP09(2012)111.

[6] CMS Collaboration, "Search for the fermiophobic model Higgs boson decaying into two photons in pp collisions at sqrt(s) $=7$ and 8 TeV", CMS PAS HIG-12-002 (2012).

[7] CMS Collaboration, "The CMS experiment at the CERN LHC", JINST 3 (2008) S08004.

[8] CMS Collaboration, "Search for a Higgs boson decaying into two photons in proton-proton collisions recorded by the CMS detector at the LHC", Phys.Lett. B710 (2012) 403-425.

[9] CMS Collaboration, "Search for the standard model Higgs boson decaying to a W pair in the fully leptonic final state in pp collisions at sqrt(s) = 7 TeV.", Phys.Lett. B710 (2012) 91-113.

[10] CMS Collaboration, "Search for the standard model Higgs boson in the decay channel $\mathrm{H} \rightarrow \mathrm{ZZ} \rightarrow 4 \mathrm{l}$ in pp collisions at sqrt(s) = 7 TeV", Phys.Rev.Lett. 108 (2012) 111804.

[11] CMS Collaboration, "Combined results of searches for the Standard Model Higgs boson in pp collisions at sqrt(s) $=7 \mathrm{TeV}$ ", Phys.Lett. B710 (2012) 26-48. 